\title{
Efecto del entrenamiento de intervalo en jugadores mexicanos juveniles de fútbol soccer \\ Effect of interval training in the performace of mexican juvenile soccer players
}

*Ricardo Ortiz-Pulido, **Rubén Ortiz-Pulido, *Julio Alejandro Gómez-Figueroa, ***Raúl Ortiz-Pulido

*Universidad Veracruzana (México), **Universidad Autónoma de Quéretaro (México), ***Universidad Autonóma del Estado de Hidalgo (México)

Resumen. En un partido de fútbol soccer los jugadores emplean su capacidad física para lograr un buen desempeño. Se ha sugerido que dicha capacidad puede ser entrenada utilizando el entrenamiento de intervalo. El objetivo de este trabajo fue comparar el rendimiento físico de jugadores juveniles de fútbol antes y después de que practicaran entrenamiento de intervalo. Para probarlo se evaluó el desempeño físico de 38 jugadores juveniles de fútbol empleando el test de pista de la Universidad de Montreal y el test de Hoff. El desempeño de los jugadores se evaluó considerando su velocidad aeróbica máxima en kilómetros por hora, el volumen máximo de oxígeno (VO2max) indirecto en mililitros por kilogramo por minuto, la distancia recorrida en metros y la frecuencia cardiaca basal en latidos por minuto en descanso, antes y después de ocho semanas de entrenamiento de intervalo. Los sujetos analizados mejoraron significativamente su capacidad aeróbica después del entrenamiento de intervalo en todas las variables medidas (velocidad aeróbica máxima $15.76 \pm 0.12$ [media \pm 1 error estándar] antes y $17.08 \pm 0.13$ después; VO2max 55.17 \pm 0.43 antes vs $59.78 \pm 0.45$ después; distancia recorrida 1461.37 \pm 11.20 antes vs $1593.79 \pm 13.21$ después; frecuencia cardiaca basal $71.58 \pm 1.14$ antes vs $67.37 \pm 1.07$ después; en todos los casos $p<0.0001)$. Concluimos que el entrenamiento de intervalo aplicado fue de ayuda para mejorar el desempeño físico de los jugadores juveniles de fútbol analizados.

Palabras clave: Entrenamiento aeróbico, jugadores de fútbol, test aeróbico, velocidad aeróbica máxima,VO2max indirecto.

Abstract. In order to achieve a good performance in a soccer match, soccer players use aerobic capacity. It has been suggested that such capacity can be trained using Interval Training. The purpose of this research was to compare the performance of juvenile soccer players before and after they practiced interval training. To prove it we assessed the physical performance of 38 juvenile soccer players using Université Montreal track test and Hoff test. Their performance was evaluated before and after the interval training measuring maximal aerobic speed in kilometers per hour, indirect maximum oxygen volume (VO2max) in $\mathrm{mm} / \mathrm{kg} / \mathrm{min}$, traveled distance in meters and basal heart rate in beats per minute. The juvenile soccer players performance increased significantly at all the variables measured after the interval training (maximal aerobic speed 15.76 \pm 0.12 [mean \pm 1 standard error] before and $17.08 \pm 0.13$ after; VO2max $55.17 \pm 0.43$ before vs $59.78 \pm 0.45$ after; traveled distance $1461.37 \pm 11.20$ before vs $1593.79 \pm 13.21$ after; basal heart rate $71.58 \pm 1.14$ before vs $67.37 \pm 1.07$ after; in all the cases $p<0.0001)$. We concluded that the interval training was helpful to improve physical performance in the juvenile soccer players tested.

Key words: aerobic training, soccer players, aerobic test maximal aerobic speed, indirect VO2max.

\section{Introducción}

El fútbol es un deporte que involucra ejercicio aeróbico (Rienzi et al., 2000), en el cual los jugadores llevan a cabo ejercicios a diferentes aceleraciones (Martínez-Cabrera et al., 2021), intensidades y pausas (Iaia et al., 2009), utilizando estrategias y planteamientos tácticos (Barrero et al., 2021). Este deporte demanda que el control motor de los jugadores sea usado a un alto nivel, requiriendo que ellos lleven a cabo habilidades técnicas y tácticas (Castañer et al., 2017), adap-

Fecha recepción: 17-06-21. Fecha de aceptación: 21-01-22

Ricardo Ortiz Pulido

pulidoortizricardo@gmail.com taciones al contexto táctico (Echavarría et al., 2021) integrándolas a patrones funcionales del juego, a traves de un sistema dinámico de movimientos y estructuras adaptadas (Ali, 2011). Para desarrollar estas habilidades y soportar un partido de fútbol con un buen rendimiento, los jugadores emplean la capacidad aeróbica (Hoff et al., 2002), considerada como una capacidad condicional que mejora el desempeño del futbolista (Castro et al., 2021), mediante la incorporación de un método que permita la cuantificación de la demanda física (Sousa et al., 2021).

La capacidad aeróbica puede ser entrenada (Impellizzeri et al., 2006) utilizando el entrenamiento de intervalo (IT, por sus siglas en inglés). El IT ha sido utilizado para evaluar el rendimiento atlético al consi- 
derarlo una medida de la capacidad aeróbica de los jugadores de fútbol (Impellizzeri et al., 2006). El IT consiste en periodos alternados de alta intensidad de entrenamiento y de descanso o pausa (Fox et al., 1997), donde el atleta entrena al 90 - 110\% de su máximo consumo de oxígeno (VO2max), medido en mililitros por kilogramos por minuto $(\mathrm{ml} / \mathrm{kg} / \mathrm{min}$ ) (Padilla et al., 1992; Billat \& Koralsztein, 1996), y de su velocidad aeróbica máxima (MAS, por sus siglas en inglés), medida en kilómetros por hora $(\mathrm{km} / \mathrm{h})$. La MAS se ha descrito como la mínima velocidad de carrera para obtener elVO2max (Di Prampero, 1986; Billat et al., 1994). A través de ella se puede controlar la intensidad del esfuerzo realizado por un jugador en un entrenamiento (Billat et al., 1995).

A pesar de que evaluar la capacidad aeróbica de un jugador es relevante para planear como mejorar su desempeño, en muchos países los clubes de fútbol donde participan jugadores juveniles no cuentan con los recursos económicos, tecnológicos y de infraestructura para hacer evaluaciones de este tipo en sus jugadores. Por ello se utilizan dos alternativas poco costosas para medir el desempeño físico de los jugadores. La primera de ellas es el test de pista de la universidad de Montréal (UMTT, por sus siglas en inglés) (Léger \& Boucher, 1980) y la segunda el test de Hoff (HT por sus siglas en inglés) (Hoff et al., 2002). Ambos son tests indirectos que permiten obtener información valiosa sobre el nivel de desempeño de los jugadores de fútbol en cualquier etapa de la temporada.

El UMTT ha sido ocupado para medir diferentes actividades en futbolistas adolescentes. Por ejemplo, para evaluar el VO2max (Bangsbo et al., 1991; Berthoin et al., 1999) y para describir la relación entre el ejercicio intermitente de alta intensidad con las velocidades finales y la intensidad del ejercicio en el juego (Dupont et al., 2008). Así mismo, ha sido ocupado para evaluar el rendimiento físico mediante la versión Vam-Eval (Mendez-Villanueva et al., 2010), que es una modificación del UMTT (Léger \& Boucher, 1980), y se han comparado sus resultados con los de un test de carrea en banda sin fin (Los Arcos et al., 2019).

El HT ha sido utilizado en el fútbol con diferentes objetivos. Por ejemplo, como ejercicio de intervalo (Hoff et al., 2002) y como forma de evaluar elVO2max (Kemi et al., 2003; Zagatto et al., 2015). Además, los resultados del HT en jugadores de fútbol se han comparado con otras pruebas, como la de intervalos de alta intensidad intermitente de ejercicio aeróbico (HIIE, por sus siglas en inghlés) (Zouhal et al., 2013), el test de ida y vuelta de 20 metros (m) y el test de Bangsbo (Nassis et al., 2010). Con el HT también se puede evaluar el sistema anaeróbico y la carrera de alta intensidad (HIR, por sus siglas en inglés) (Chamari et al., 2005; Loures et al., 2015; Zagatto et al., 2016).

A pesar de que el UMTT y el HT miden la capacidad aeróbica de los futbolistas (Ferreira et al., 2011; Loures et al., 2015; León et al., 2015; Zagatto et al., 2016; Bangsbo et al., 1991; Berthoin et al., 1999), hasta la fecha no existe ninguna investigación publicada que describa el efecto simultáneo del IT en los valores obtenidos con UMTT y HT.

Por lo anterior, en este trabajo usamos al UMTT y al HT para evaluar la mejora en el desempeño físico en jugadores juveniles de fútbol que usan IT en su entrenamiento. El UMTT lo ocupamos para obtener la MAS y el VO2max indirecto, el HT lo usamos para medir la distancia recorrida de cada jugador, y ambas pruebas para obtener la frecuencia cardiaca basal. Ello nos permitió comparar el rendimiento de los jugadores en dos momentos de su entrenamiento, antes y después de la aplicación del IT. Hipotetizamos que, si el IT favorece el desempeño físico de los jugadores de fútbol juveniles, el IT aplicado al entrenamiento de fútbol de jugadores juveniles durante ocho semanas mejorará su MAS, la frecuencia cardiaca basal, el VO2max indirecto y la distancia recorrida.

\section{Material y método}

La investigación se llevó a cabo en dos diferentes temporadas 2010-2011 y 2013-2014 en los meses de junio, julio y agosto. Los futbolistas residieron en el momento del estudio en Xalapa, Veracruz, México (ciudad ubicada a 1460 metros sobre el nivel del mar; en las coordenadas $19^{\circ} 32^{\prime}$ al Norte y $96^{\circ} 54^{\prime}$ al Oeste). Los participantes iniciales del estudio fueron 49 jugadores juveniles (menores de 19 años) de fútbol del equipo Búhos de la tercera división de fútbol mexicano. La muestra final quedó en 38 jóvenes después de descontar a aquellos individuos que no cubrieron los siguientes criterios de inclusión: tener al menos un año de experiencia en el nivel de tercera división profesional, no haber practicado previamente el IT (al menos cuatro meses antes de iniciar el estudio) y participar en el 100\% de las sesiones de entrenamiento. En ambas temporadas se excluyó de la muestra a cuatro sujetos que no realizaron el total de las evaluaciones, tres que se ausentaron del entrenamiento, tres que se lesionaron y uno que se enfermó. Las características físicas medidas 
de los jugadores, que no variaron significativamente antes y después de las ocho semanas de IT, se indican en la Tabla 1.

Los participantes y sus padres fueron informados sobre el protocolo de prueba seguido en este estudio. Los padres revisaron y firmaron un formulario de consentimiento voluntario que siguió los lineamientos de la declaración de Helsinki. Antes del estudio, los participantes jugaban al fútbol dos días a la semana durante dos meses, participando en partidos amistosos en su club. En estas ocasiones jugaron dos mitades de 40 minutos, con calentamiento previo y estiramiento de 20 minutos; durante este periodo no existió un entrenamiento físico. El equipo no tuvo partidos oficiales durante el período de estudio.

Los sujetos fueron evaluados por el equipo médico del Club de fútbol quienes verificaron que no tuvieron lesiones y que pudieran participar en el programa de entrenamiento aeróbico. A los sujetos se les informó que podían abandonar el estudio en cualquier momento y que no podrían ser identificados en los reportes que resultaran del mismo. El protocolo de estudio fue aprobado por el comité de ética del equipo.

\section{Entrenamiento}

El entrenamiento duró 14 semanas y su programación puede ser consultada en las Tablas 1, 2 y 3 . En resumen, los sujetos entrenaron cinco días por semana de lunes a viernes, con un tiempo de entrenamiento diario que duró entre 90-150 minutos, tiempo que varió según las pausas hechas para organizar las actividades. Los sujetos hicieron IT solo martes y jueves. Todas las sesiones de entrenamiento se realizaron entre las 1600-1830 hrs. Durante las primeras cuatro semanas del entrenamiento, antes de iniciar la temporada oficial de fútbol mexicano de tercera división, los sujetos fueron familiarizados con las pruebas UMTT y HT; para ello se les enseño como tomarse la frecuencia cardíaca (HR, por sus siglas en inglés) manualmente con reloj (Ortiz-Pulido et al., 2018) y a registrar el índice de esfuerzo percibido (RPE, por sus siglas en inglés) (Borg, 1970). El RPE mide el esfuerzo auto-percibido mediante una calificación que el sujeto otorga en una escala de seis a 20, donde el número seis indica que el esfuerzo fue ligero y el número 20 indica que fue extremadamente fuerte. El adiestramiento para toma de la HR se realizó durante las primeras cuatro semanas del programa, los martes y jueves.

Para familiarizar a los jugadores con las pruebas UMTT y HT se les adiestró en ellas los martes y jue- ves, respectivamente, de las primeras cuatro semanas del programa de entrenamiento IT (Tabla 1). Por ejemplo, el martes de la semana uno, al practicar UMTT los sujetos recorrieron en dos minutos, a una velocidad de ocho km/hr, $280 \mathrm{~m}$ de una pista atlética de tartán (ver Tabla 1); posteriormente se les indicó en qué consistía la evaluación de la prueba UMTT (descripción abajo). Una vez hecho esto se les pidió que realizarán un ejercicio ligero.

En cuanto al HT, como ejemplo, el jueves de la semana uno, los sujetos practicaron el HT con un esfuerzo ligero. Para ello los sujetos recorrieron (caminando) dos veces un circuito de HT marcado de 290 m en un campo de fútbol (ver detalles más adelante). Cada jugador en la primera vuelta caminó a través del circuito; en la segunda vuelta recibió un balón que pasó por todo el circuito de forma libre; posteriormente se les indicó en qué consistía la prueba de HT. Una vez terminada la explicación se les pidió que realizaran un ejercicio ligero (ver Tabla 1).

En las semanas dos a la 14, salvo cuando hicieron las pruebas UMTT y HT, al iniciar su entrenamiento los sujetos realizaron un calentamiento previo con una duración aproximada de 20-30 minutos controlado y dirigido por el preparador físico del equipo. El entrenamiento previo consistió en: ocho ejercicios de estiramientos asistidos de flexión y extensión del tren inferior con una duración de 15 segundos de aplicación por 15 segundos de pausa; $1000 \mathrm{~m}$ de trote suave a 66:30 minutos $/ \mathrm{km}$; cuatro ejercicios específicos de fútbol, dos sin balón y dos con balón, de un minuto de duración cada uno (estos ejercicios se llevaron a cabo en un cuadrado de dos por dos metros, delimitado por conos, donde al inicio los jugadores se ubicaron en el centro del cuadro y a una señal corrieron de frente hacia uno de los conos, le dieron la vuelta y regresaron a la posición de inicio y posteriormente lo hicieron con los tres conos restantes); dos series de cinco, siete y $10 \mathrm{~m}$ de aceleraciones cortas (la primera serie a submáxima velocidad y la otra a máxima velocidad, con pausa de 30 segundos entre cada aceleración y tres minutos entre las series) y, finalmente; cuatro ejercicios de estiramiento asistido del tren inferior con una aplicación 30 segundos por 30 segundos de pausa. Una vez concluido este calentamiento los sujetos realizaron la programación descrita en la Tabla 1.

Los jugadores entrenaron IT de la semana seis a la 13. La evaluación en las pruebas UMTT y HT se realizaron martes y jueves en las semanas cinco y 14, es decir; antes y después de IT, respectivamente. Antes de 
las pruebas, los jugadores de fútbol hicieron 20 minutos del calentamiento descrito en el párrafo previo, pero eliminado los ejercicios específicos de futbol. Una vez que los individuos estuvieron listos realizaron las pruebas. Los datos obtenidos por sujeto en el UMTT fueron MAS y V02max indirecto considerando la última etapa de dos minutos completada; mientras que en el HT se registró la distancia máxima recorrida en metros.

En función de su MAS inicial obtenida, cada jugador de fútbol siguió un programa de entrenamiento de la semana seis a la 13 (ver más abajo). El programa de IT que siguió cada jugador de fútbol consistió en un entrenamiento aeróbico de 100\% de MAS en las distancias propuestas.

\section{Instrumentos}

\section{Université de Montréal track test}

Este es un método indirecto continuo de etapas múltiples máximas, propuesto por Léger \& Boucher (1980). En el presente estudio el sujeto comenzó a caminar o correr a una velocidad inicial de siete $\mathrm{km} / \mathrm{h}$ a lo largo de una pista de tartán de $400 \mathrm{~m}$, cada dos minutos su velocidad aumentó en un $\mathrm{km} / \mathrm{h}$. Una señal auditiva producida por un reproductor de cinta ubicado en el centro del óvalo de la pista indicó a los participantes cuándo deberían aumentar su velocidad. La prueba finalizó cuando el sujeto no pudo recorrer la distancia estipulada en al menos dos metros. En nuestro caso, controlamos la velocidad de los jugadores de fútbol colocando conos rojos cada 20 metros a lo largo de la pista, para que cada sujeto pudiera regular su velocidad de acuerdo con el sonido reproducido, y llegar al siguiente cono a tiempo. Registramos la MAS $(\mathrm{km} / \mathrm{h})$ y el VO2max indirecto durante el UMTT para cada sujeto. La MAS se calculó utilizando la siguiente fórmula (Kuipers et al., 1985): MAS $=\mathrm{Sf}+(\mathrm{t} / 60 \cdot 0.5)$, donde la velocidad final (Sf por sus siglas en inglés), fue la ultima velocidad completada en $\mathrm{km} / \mathrm{h}$ y t el tiempo en segundos de la etapa incompleta. El VO2max se obtuvo mediante la siguiente formula: $\mathrm{VO} 2 \mathrm{max}=3.5(\mathrm{v})$; donde $\mathrm{v}=$ velocidad obtenida del periodo completado (Léger \& Mecier, 1984). En el caso de que los jugadores no completaran un minuto de la ultima etapa se mantuvo la MAS que lograron completar.

\section{Prueba de Hoff}

Este es un método propuesto por Hoff et al. (2002) donde el ejercicio específico de fútbol puede ser realizado con balón y usarse como entrenamiento de inter- valo aeróbico. Los detalles y la descripción del HT pueden ser consultados en diversas publicaciones (Hoff et al., 2002; Kemi et al., 2003; Chamari et al., 2005; McMillan et al., 2005; Hoff, 2005) y en la Fig. 1. En nuestro estudio el sujeto condujo un balón durante todo el HT. Durante la prueba el jugador repitió este circuito tantas veces como pudo durante 10 minutos. Al final se registró la distancia en metros recorrida para cada jugador de fútbol.

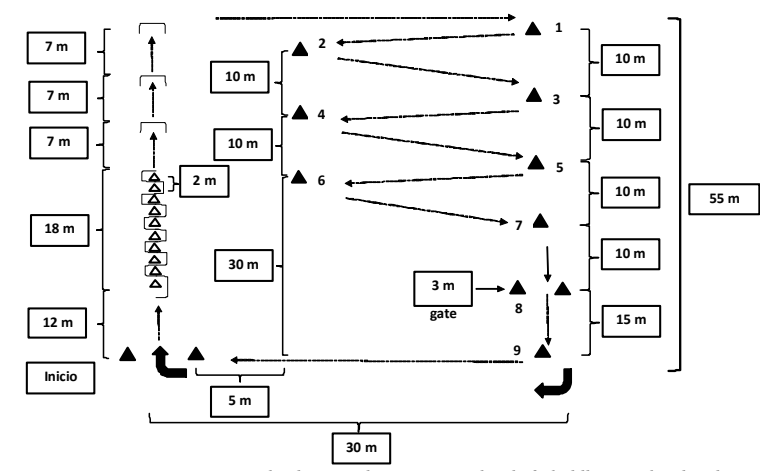

Figura 1. Esquema representando el recorrido que un jugador de futbol lleva a cabo al realizar el HT. El jugador comienza la prueba en donde dice inicio (en la esquina inferior izquierda) corriendo hacia el frente $10 \mathrm{~m}$, enseguida realiza movimientos de zig-zag durante $18 \mathrm{~m}$ entre conos separados colocados en línea recta cada dos $\mathrm{m}$. Posteriormente el jugador salta tres obstáculos de $30 \mathrm{~cm}$ de altura que están ubicados en línea recta a siete metros de distancia cada uno durante $21 \mathrm{~m}$. A continuación, una vez terminando de saltar el último obstáculo, el jugador se dirige hacia un cono ubicado a $36 \mathrm{~m}$, en esta parte el jugador hace zig-zag lo más rápido posible entre seis conos colocados en dos líneas paralelas (la separación entre sus líneas rápido posible entre seis conos colocados en dos líneas paralelas (la separacion entre sus línes
es de $25 \mathrm{~m}$ ). Enseguida el jugador avanza hacia atrás $10 \mathrm{~m}$ hasta donde está marcado con dos conos. Posteriormente, avanzando de frente recorre $15 \mathrm{~m}$ y gira para continuar $30 \mathrm{~m}$ más de distancia hasta el final del circuito, reiniciando el recorrido otra vez.

La prueba fue llevada a cabo hasta con cinco jugadores simultáneamente en el campo, separados entre sí por intervalos de 10 segundos. De forma verbal a cada jugador se le indicó el inicio de la prueba (momento en que se le inició a cronometrar el tiempo), la mitad de la prueba (cuando llegó al minuto cinco) y la cercanía del final de la prueba (cuando le faltaba un minuto para finalizar). Durante toda la prueba se motivó verbalmente a los jugadores.

\section{Aplicación de las pruebas}

Las pruebas UMTT y HT fueron realizadas con 48 horas de diferencia. Una hora antes de iniciar las pruebas se pidió a cada jugador que midiera manualmente su HR basal. La HR máxima (HRmax, por sus siglas en inglés) se midió por un asistente médico para cada participante al finalizar cada prueba. Cada asistente médico fue previamente capacitado para la toma de dicha medida en 15 segundos, contando con un cronometro y un formato de registro con la lista de los participantes.

Antes de cada prueba los participantes descansaron dos días. Este periodo descanso consistió en que hicieron cargas de entrenamientos reducidas, tanto en intensidad como en volumen; además, no ingirieron comidas durante al menos 3 horas antes de cada prueba. 
Tabla 1

Adiestramiento individual en las pruebas (a) UMTT y (b) HT aplicado en este estudio a 38 jugadores de fútbol juvenil mexicanos de la tercera división profesional. El adiestramiento lo recibieron durante las cuatro semanas iniciales del programa, dedicando los martes a UMTT y los jueves a HT.

\begin{tabular}{|c|c|c|c|c|c|c|c|}
\hline \multirow{2}{*}{ Prueba } & \multirow[b]{2}{*}{ Día/semana } & \multicolumn{2}{|r|}{ Repeticiones } & \multirow{2}{*}{ Distancia recorrida $(\mathrm{m})$ por repetición } & \multirow{2}{*}{ Intensidad de carrera $(\mathrm{km} / \mathrm{h})$} & \multirow{2}{*}{$\begin{array}{c}\text { Distancia total }(\mathrm{m}) \\
\text { recorrida por cada jugador }\end{array}$} & \multirow{2}{*}{$\begin{array}{c}\text { Pausa entre repeticiones } \\
\text { (minutos) }\end{array}$} \\
\hline & & Número & Tiempo (minutos) repetición & & & & \\
\hline \multirow[t]{3}{*}{ (a) UMTT } & Martes/semana 1 & 1 & $2^{\prime}$ & 280 & 8 & 280 & ad libitum \\
\hline & Martes/semana 2, 3 & 2 & $2^{\prime}$ & 280,320 & 8,9 & 600 & ad libitum \\
\hline & Martes/semana 4 & 3 & $2^{\prime}$ & $280,320,320$ & $8,9,10$ & 920 & ad libitum \\
\hline \multirow[t]{3}{*}{ (b) $\mathrm{HT}$} & Jueves / semana 1 & 1 & ad libitum & 290 & Ligera & 290 & \\
\hline & Jueves/semana 2, 3 & 2 & ad libitum & 290,290 & Ligera & 580 & $3^{\prime}-5{ }^{\prime}$ \\
\hline & Jueves/semana 4 & 3 & ad libitum & $290,290,290$ & Ligera & & $3^{\prime}-5{ }^{\prime}$ \\
\hline
\end{tabular}

\section{Tabla 2.}

Esquema general de entrenamiento semanal aplicado durante este estudio a 38 jugadores de fútbol juveniles mexicanos de la tercera división profesional. El calentamiento general se hizo durante todas las semanas del estudio, el entrenamiento especifico de la semana 6 a la 13.per

Día Calentamiento General Entrenamiento específico

Lunes Cuatro ejercicios de Tres series de carrera de tres distancias (3, 5 y $7 \mathrm{~m}$ ) a MAS con pausa entre distancias de 30" y pausa entre series de 3'; IT (ver Tabla 3), y; cinco series de ejercicios estiramiento sin asistencia asistidos de flexibilidad del tren inferior, con 12-15 repeticiones por ejercicio, con pausa de 15" entre ejercicios y 30" entre series. y cuatro ejercicios de Series de carrera de velocidad de tres distancias $(10,20$ y $30 \mathrm{~m}$ ) a MAS, cinco series hechas por los defensas y delanteros y tres llevadas a cabo por los medios, con estiramiento con asistencia, pausa de 1' entre distancias y 5' entre series; ejercicios técnicos de fútbol individual, que consistieron en dominio de balón (con pies, músculos del cuádriceps y donde el sujeto se mantuvo cabeza), conducción de balón, pases cortos a 2 y 3 m con un companero, cada ejercicio tuvo una duración de 5' y al cambiar de ejercicio se ejecutó una pausa de 2'; de pie realizando una cinco series de cinco prácticas personalizadas en equipo, que se llevaron a cabo colocando a los 11 jugadores en su posición en el terreno de juego y sé les indico que

Martes flexión del tronco inferior realizaran movimientos propios de la posición durante 15” descansaran 10” y repitieran hasta cinco veces el proceso, con pausa de 1' entre series; recorrido de $2 \mathrm{~km}$ a y piernas juntas, las manos una velocidad de 6'30" por km; series de 20 repeticiones de ejercicios de fuerza abdominal, que se llevaron a cabo haciendo 3, 4, 5 y 6 series las semanas seis, siete, trataran de tocar las puntas ocho y nueve, respectivamente, con pausa de 30" entre serie; series de 15 repeticiones de ejercicios de fuerza de media sentadilla, realizando 2,3 y 4 series en las de los pies (30" de semanas seis, siete y ocho, respectivamente, con pausa de 30" entre serie, y; cuatro ejercicios asistidos de estiramiento con flexión y extensión del tronco inferior, aplicación por 15” de con 15” de aplicación por 15” de pausa.

pausa); Carrera ligera $\quad$ Cinco carreras de $100 \mathrm{~m}$ corriendo al 100\% de MAS; IT (ver Tabla 3); cuatro estiramientos sin asistencia de flexión del tronco con extensión de piernas juntas, con (6'00"- 6'30" el 10 " de aplicación por 1' entre estiramientos, y; cuatro estiramientos con asistencia de flexión del tronco con extensión de piernas separadas en la posición de sentado, kilómetro); los defensas, 10 "de aplicación con un período de recuperación de 1' entre estiramientos.

$\begin{array}{ll}\text { kilómetro); los defensas, } & 10 \text { " de aplicación con un período de recuperación de 1' entre estiramientos. } \\ \text { porteros y delanteros } & \text { Seis series de técnica básica de vallas con } 10 \text { vallas (cada valla tuvo una altura de } 78 \mathrm{~cm} \text { y estuvo separada por } 1 \mathrm{~m} \text { de distancia de otra valla), que se realizó indicando }\end{array}$ realizaron $2 \mathrm{~km}$ y $3 \mathrm{~km}$ los a los sujetos que se ubicara de frente a la valla inicial, una vez estando ahí se les pidió que elevaran una de sus piernas para superar la valla y enseguida hacer lo mismo medios; Entrenamiento de con la pierna de atrás, con pausa de 1' entre series; carrera de velocidad al $100 \%$ de MAS, recorriendo tres veces $40 \mathrm{~m}$, dos $50 \mathrm{~m}$ y una $60 \mathrm{~m}$, con 1' de descanso técnica de carrera, entre carreras; series de técnica de fútbol individual, que consistió en dar 10 pases rasos a un compañero que se encontró a $5 \mathrm{~m}$, repitiendo el mismo proceso a 10 , haciendo cinco series de 15 y $20 \mathrm{~m}$; partido amistoso, con dos mitades de 40' cada una; series de 20 repeticiones de ejercicios de fuerza abdominal, que se llevaron a cabo haciendo 3, 4, 5 y 6 tres ejercicios cada una, los series las semanas 11,12, 13 y 14, respectivamente, con pausa de 30" entre serie; series de 15 repeticiones de ejercicios de fuerza de media sentadilla, realizando 2, 3 ejercicios fueron elevación y 4 series en las semanas 9,10 y 11, respectivamente, con pausa de 30 ” entre serie, y; cuatro ejercicios asistidos de estiramiento con flexión y extensión del tronco $\begin{array}{ll}\text { ejercicios fueron elevación } & \text { y } 4 \text { series en las semanas } 9,10 \text { y } 11, \text { respectivas } \\ \text { de rodillas, paso yogi y } & \text { inferior, con } 15 \text { " de aplicación por 15" de pausa. }\end{array}$

$\begin{array}{ll}\text { de rodillas, paso yogi y } & \text { inferior, con 15” de aplicación por 15” de pausa. } \\ \text { golpeando el talón al } & 1 \mathrm{~km} \text { de carrera controlada a 6’00"- 6’30" por } \mathrm{km} \text {; seis series de 1' de ejercicios técnicos de fútbol individual, que consistieron en conducir tres veces el balón con el }\end{array}$ glúteo, mismos que fueron pie derecho y tres veces con el pie izquierdo en un cuadrado de 2 x $2 \mathrm{~m}$ que fue delimitado por conos, al inicio los jugadores tomaron la posición en el centro del hechos en un tramo de 20 cuadro y a una señal condujeron el balón con la pierna derecha hacia uno de los conos, le dieron la vuelta y regresaron a la posición de inicio para posteriormente Viernes $\mathrm{m}$, regresando caminando, hacer lo mismo con los tres conos restantes, con pausa de 1', para posteriormente usar la otra pierna; $1 \mathrm{~km}$ de carrera controlada a 5'00”-5' 30 " por $\mathrm{km}$ sin pausas entre series. El entrenamiento estratégico, que tuvo una duración de 60' y fue realizado en el campo de fútbol, donde el entrenador explicó y detalló cada una de las estrategias que calentamiento duró en planeadas para el juego; dos ejercicios de flexibilidad sin asistencia y cuatro ejercicios con asistencia, donde el sujeto se mantuvo de pie realizando un estiramiento promedio de $42^{\prime} \pm 23^{\prime} 30^{\prime}$. con flexión y del tronco inferior con las piernas juntas y donde las manos trataron de tocar las puntas de los pies, con 15” de aplicación por 15” de pausa. $\frac{\text { promedio de } 42^{\prime} \pm 23^{\prime} 30^{\prime \prime} \text {. con flexión y del tronco }}{\text { IT entrenamiento de intervalo. MAS velocidad aeróbica máxima. }}$

Tabla 3

Programa de IT semanal (semanas 6 a 13) aplicado durante este estudio a 38 jugadores de fútbol juvenil mexicanos de la tercera división profesional.

\begin{tabular}{|c|c|c|c|c|c|}
\hline Semana & \# de repeticiones & $\begin{array}{l}\text { Distancia recorrida }(\mathrm{m}) \text { por } \\
\text { repetición }\end{array}$ & $\begin{array}{l}\text { Intensidad de } \\
\text { carrera (MAS) }\end{array}$ & $\begin{array}{l}\text { Distancia total }(\mathrm{m}) \\
\text { recorrida por cada jugador }\end{array}$ & Pausa entre repeticiones (minutos) y forma de la recuperación \\
\hline Semana 6 & 3 & $500,500,500$ & $100 \%$ & 1500 & 1'30" caminando y 4' de flexibilidad \\
\hline Semana 7 & 4 & $500,500,500,500$ & $100 \%$ & 2000 & 1'30" caminando y 4' de ejercicios de flexibilidad \\
\hline Semanas 8,9 & 4 & $500,1000,500,1000$ & $100 \%$ & 3000 & $\begin{array}{l}\text { Después de } 1^{\mathrm{a}} \text { repetición } 1^{\prime} 30^{\prime \prime} \text { técnica de futbol; después de } 2^{\mathrm{a}} \text { y } 3^{\mathrm{a}} \text { repetición } 1^{\prime} 30^{\prime \prime} \\
\text { caminando y } 4^{\prime} \text { de ejercicios de flexibilidad, y; después de } 4^{\mathrm{a}} \text { repetición } 1^{\prime} 30 \text { " caminando }\end{array}$ \\
\hline Semanas 10,11 & 4 & $1000,1000,1000,1000$ & $100 \%$ & 4000 & 4' de carrera al $70 \%$ de MAS \\
\hline Semana 12 & 6 & $500,500,500,500,500,500$ & $100 \%$ & 3000 & $\begin{array}{l}\text { Después de la } 1^{a}, 2^{a} \text { y } 3^{a} \text { repetición } 2^{\prime} \text { de carrera al } 70^{\%} \text { de MAS, } 2 \text { series de técnica de futbol } \\
\text { de } 1^{\prime} \text { y } 1^{\prime} \text { de ejercicios de flexibilidad; después de la } 4^{a}, 5^{a} \text { y } 6^{a} \text { repetición } 2^{\prime} \text { de carrera al } 70 \% \\
\text { de MAS }\end{array}$ \\
\hline
\end{tabular}

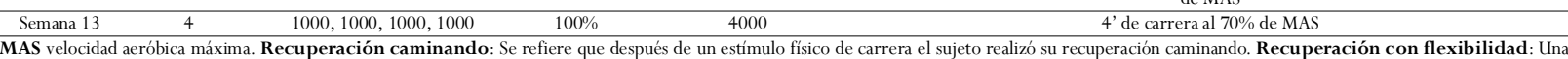
MAS velocidad aeróbica máxima. Recuperación caminando: Se refiere que después de un estímulo físico de carrera el sujeto realizó su recuperación caminando. Recuperación con flexibilidad: Una
vez finalizado el estímulo físico el sujeto realiź su recuperación con ejercicios de flexibilidad, para ello el sujeto realizó uno o cuatro ejercicios sin asistencia dependiendo de lo indicado en la Tabla 3 , e ejercicio consistió en que el futbolista se sentará en una superficie plana con las piernas en extensión realizando un estiramiento con flexión del tronco al frente, piernas juntas y con las manos tratando de tocar las puntas de los pies, con 30 segundos de aplicación por 30 segundos de pausa. Recuperación con técnica de fútbol: Al término de la carrera el sujeto inmediatamente realizó ejercicios de técnica de fútbol. Estas series se llevaron a cabo en un área a $10 \mathrm{~m}$ de distancia de donde el sujeto finalizó su carrera. En esa área se colocaron conos de $20 \mathrm{~cm}$ de diámetro y $10 \mathrm{~cm}$ de altura en dos líneas paralelas a $2 \mathrm{~m}$ de distancia entre ellas. Allí los sujetos se agruparon por parejas y tomaron un balón (del número cinco), ubicando a los jugadores atrás de los conos y frente a frente (es decir, hubo una distancia de $2 \mathrm{~m}$ entre los jugadores); entonces realizaron ejercicios. Una serie de estos ejercicios consistió en: 10 pases de balón dirigiéndolo a su compañero con la parte interna del pie derecho y después 10 con el pie izquierdo, para enseguida hacer 10 pases con la parte externa del pie derecho y después 10 con el pie izquierdo. Recuperación al 70\% de la MAS: Al finalizar el estímulo físico de $100 \%$ de carrera de MAS en la distancia propuesta, el sujeto disminuyó su intensidad de carrera al 70\% de su MAS durante el tiempo propuesto que se les indico para la recuperación.

El día de la prueba, la evaluación de la HR basal inicio a las 15:00 p.m., para ello se pidió a los sujetos que se recostaran en el suelo del campo de fútbol durante 30 minutos, donde permanecieron quietos en una posición supino. Al termino de los 30 minutos se les pidió que realizaran la medición, siguiendo la metodología ya conocida (Ortiz-Pulido et al., 2018). Los datos tomados fueron visualmente inspeccionados por los asistentes médicos buscando errores y, si no los hubo, fueron registrados.

Después de cinco minutos de terminadas las pruebas (UMTT y HT), se pidió a los participantes que indi- caran el esfuerzo realizado utilizando el RPE (Borg, 1970). Todos los participantes reportaron el RPE. Para corroborar si el esfuerzo percibido se reflejaba físicamente, este fue comparado con la HRmax registrada al final de cada uno de los test realizados. Comparando RPE y HRmax comprobamos que efectivamente cada individuo participante había hecho su mejor esfuerzo durante ambas pruebas.

Para medir la HRmax al terminar cada prueba se usó el siguiente procedimiento: en la UMTT los asistentes médicos se ubicaron a lo largo de la pista, cada 20 m y tomaron la HRmax inmediatamente después de 
que observaron que algún futbolista abandono la UMTT; en el HT, se asignó a cada futbolista un asistente, dicho asistente observó el desempeño del jugador y en el último minuto de la prueba corrió a tres metros de distancia de él, registrando la HRmax en el momento en que el jugador terminó la prueba.

\section{Análisis estadístico}

Los datos obtenidos para cada variable fueron evaluados para detectar desviaciones de la normalidad usando una prueba de Shapiro-Wilk. Para determinar si existieron diferencias entre cada variable antes y después del IT, en caso de detectar una distribución normal, se usó una prueba de $t$ de Student pareada (t), considerando como datos pareados a los tomados a cada sujeto de estudio antes y después del IT; sí, por el contrario, se detectó una distribución no normal, se usó una prueba de Mann-Whitney (U). En todos los casos se consideró una $p<0.05$ para rechazar la hipótesis nula, se usó el programa PAST 3.5 (Hammer et al., 2001) y se reportan los valores como media \pm 1 error estándar (ee). Como resultado de las pruebas estadísticas llevadas a cabo se indica su acrónimo, U ó t, los grados de libertad o tamaño de muestra (gl ó n) y el alfa (p).

\section{Resultados}

El desempeño físico de los participantes fue diferente antes y después del IT. Así, los jugadores evaluados presentaron valores significativamente diferentes antes y después de la aplicación del IT (Tabla 4) en su MAS $\left(\mathrm{U}=197.5, \mathrm{n}_{1}=38, \mathrm{n}_{2}=38, p<0.0001\right), \mathrm{VO} 2 \max (\mathrm{U}$ $\left.=197.5, \mathrm{n}_{1}=38, \mathrm{n}_{2}=38, p<0.0001\right)$, distancia recorrida $(\mathrm{t}=-19.20, \mathrm{gl}=37, p<0.0001)$ y frecuencia cardiaca basal $\left(\mathrm{U}>496.4, \mathrm{n}_{1}=38, \mathrm{n}_{2}=38, p<0.01\right)$. La MAS, el VO2max y la distancia recorrida fueron

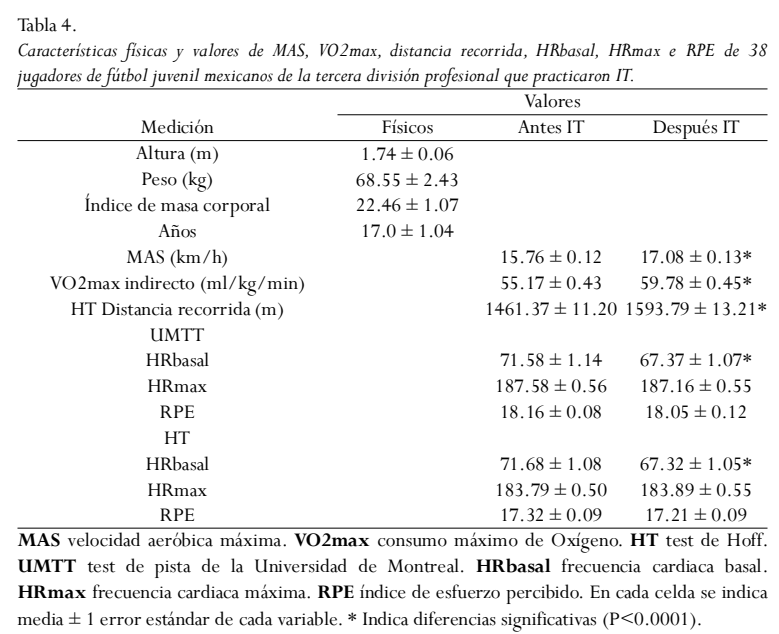

mayores después de la IT que antes (Tabla 4), mientras que la frecuencia cardiaca basal fue menor. En la distancia recorrida los jugadores juveniles de fútbol mejoraron nueve por ciento al realizar el IT.

\section{Discusión}

En jugadores de fútbol juveniles se ha descrito una relación positiva entre la capacidad aeróbica (Hoff et al., 2002) y el rendimiento específico en los partidos de fútbol (Impellizzeri et al., 2006). Dado que se ha demostrado que la aplicación del IT en jugadores de fútbol ha permitido mejorar su capacidad aeróbica (Chamarri et al., 2005) y su rendimiento deportivo de alto nivel durante los partidos de fútbol (Stølen et al., 2005), en este estudio probamos si este efecto ocurría también en jugadores de fútbol juveniles.

Nuestros resultados indican que ocho semanas de IT contribuyeron a mejorar la MAS, el VO2max indirecto, la distancia recorrida y la frecuencia cardiaca basal de los sujetos analizados. Nuestro estudio coincide con los resultados obtenidos por otros investigadores que usaron el IT para mejorar el rendimiento en jugadores de fútbol (Helgerud et al., 2001; McMillan et al., 2005; Sassi et al., 2005; Impellizeri et al., 2006; Bravo et al., 2008). Por lo que sabemos, esta es la primera vez que la influencia del IT en el UMTT y HT se ha medido simultáneamente en jugadores juveniles de fútbol soccer.

\section{Efectos en MAS}

En equipos de fútbol que tienen altas posibilidades económicas, la MAS puede evaluarse a través de variables que se miden con herramientas tecnológicas costosas (Rampinini et al., 2007; Bravo et al., 2008; Dupont et al., 2008; Iaia et al., 2009; Buchheit et al., 2010b). Este es el caso de la medición de la HIR (High Intensity Runing) (Rampinini et al., 2007; Bravo et al., 2008; Dupont et al., 2008) y de la VHIA (Very High-Intensity Activities) (Rampinini et al., 2007). Sin embargo, los valores del HIR mayores a $15 \mathrm{~km} / \mathrm{h}$ (Iaia et al. 2009) y los de VHIA $>16.1 \mathrm{~km} / \mathrm{h}$ (Buchheit et al., 2010b) son muy cercanos a los valores de MAS evaluada por el UMTT (Léger \& Boucher, 1980; Cazorla, 1990; Dupont et al, 2004, 2008; Carminatti et al., 2013), lo que abre la posibilidad de usar la UMTT como una opción menos costosa.

Nosotros encontramos una mejora significativa de la MAS después de la aplicación de IT en jugadores de fútbol juveniles. Esto también ha sido reportado por otros autores (Chamari et al. 2005; Santos-Silva et al., 
2007; Carbonel et al., 2009; Buchheit et al. 2010a, 2010b; Gomez-Piqueras et al., 2010; Calahorro et al., 2012; Buchheit \& Mendez-Villanueva 2013; Los Arcos et al., 2019). Entre todos ellos es interesante el reporte de Paul et al. (2019) quienes señalan que encontraron una mejora significativa en la MAS con solo cuatro semanas de entrenamiento de IT, lo que abre la posibilidad de que con un entrenamiento menor a ocho semanas los jugadores pueden mejorar su desempeño.

Por ello, al reflexionar sobre estos aspectos, hemos considerado una aplicación práctica para entrenar la intensidad de la MAS cuando no existan posibilidades de conocer los valores de HIR ó VHIA. Nuestra sugerencia consiste en entrenar el IT mediante cambios de dirección tomando en cuenta a la MAS de cada sujeto de acuerdo con la posición que ocupa en el campo de fútbol, el nivel competitivo y edad cronológica (Buchheit et al., 2010b), considerando para ello los estudios previos realizados del volumen total de HIR ó VHIA que han sido descritos en un juego de fútbol (Buchheit et al., 2010a; Mendez-Villanueva et al., 2013). De esta forma, se podría obtener las demandas físicas y fisiológicas acordes a la exigencia del juego del fútbol y lograr un efecto óptimo en ese deporte (Helgerud et al., 2001). Por tanto, consideramos que el IT puede utilizarse dentro de los diferentes periodos del entrenamiento de futbolistas para mejorar su rendimiento.

\section{Efectos en V02max}

Diferentes autores (Helgerud et al., 2001; McMillan et al., 2005; Chamari et al., 2005) obtuvieron un incremento del V02max antes y después $(58.1 \pm 4.5 \mathrm{ml} /$ $\mathrm{km} / \mathrm{min}$ vs $64 \pm 3.9 \mathrm{mil} / \mathrm{kg} / \mathrm{min} ; 63.4 \pm 5.6 \mathrm{ml} / \mathrm{kg} /$ $\min$ a $69.8 \pm 6.6 \mathrm{ml} / \mathrm{kg} / \mathrm{min} ; 65.3 \pm 5.0 \mathrm{ml} / \mathrm{kg} / \mathrm{min}$ vs $70.7 \pm 4.3 \mathrm{ml} / \mathrm{kg} / \mathrm{min}$ ) de aplicar el IT en ocho y 10 semanas; con jugadores de diferentes años de edad (de 14 a 18 años). Así mismo, Impellizeri et al. (2006) evidencia el efecto del entrenamiento de IT en dos grupos de entrenamiento genérico $(n=15)$ y entrenamiento específico aeróbico $(n=14)$; en jugadores de fútbol de $17.2 \pm 0.8$ años de edad, cuando los dos grupos fueron testados en VO2max previamente $(55.6 \pm 3.4 \mathrm{ml} / \mathrm{kg} /$ $\min$ vs $57.7 \pm 4.2 \mathrm{ml} / \mathrm{kg} / \mathrm{min}$ ), después de cuatro (59.7 $\pm 4.1 \mathrm{ml} / \mathrm{kg} / \mathrm{min}$ vs $60.2 ; \pm 3.9 \mathrm{ml} / \mathrm{kg} / \mathrm{min}$ ) y ocho semanas $(61.4 \pm 4.6 \mathrm{ml} / \mathrm{kg} / \mathrm{min}$ vs $61.8 \pm 4.5 \mathrm{ml} /$ $\mathrm{kg} / \mathrm{min}$ ) en una banda sin fin en el laboratorio. En nuestro estudio también existió un aumento de VO2max indirecto a finalizar las ocho semanas.

Al comparar nuestra investigación con Aziz et al. (2008) en jóvenes futbolistas de16 y 18 años de edad, quienes fueron evaluados por medio del test de ida $\mathrm{y}$ vuelta, encontramos que existió un V02max (51.2 \pm $3.5 \mathrm{ml} / \mathrm{kg} / \mathrm{min}$ ) menor al obtenido por nosotros. También Barrera et al. (2021) obtuvo datos similares de V02max con los jóvenes futbolistas chilenos menores de 14 años de edad, sin embargo, en el mismo estudio describió los datos del VO2max en los jóvenes menores de 15,16, 17, 18 y 19 años de edad, los cuales son muy parecidos a los nuestros.

Otros trabajos que tuvieron mucha similitud con nuestros resultados, pero que no evaluaron el efecto del IT, fueron los siguientes: Aziz et al. (2005) evidenció sus resultados del V02max de jóvenes futbolistas (17.7 \pm 0.4 de años de edad) en tres diferentes test (MST: 59.1 $\pm 4.8 \mathrm{ml} / \mathrm{kg} / \mathrm{min} ;$ YIET: $56.1 \pm 4.5 \mathrm{ml} / \mathrm{kg} / \mathrm{min}$; TRT: $57.8 \pm 5.0 \mathrm{ml} / \mathrm{kg} / \mathrm{min}$ ); Chamari et al. (2005) también describió el V02max en jugadores juveniles de fútbol (61.1 $\pm 4.6 \mathrm{ml} / \mathrm{kg} / \mathrm{min})$; igual que Cunha et al. (2011) $(59.6 \pm 4.3 \mathrm{ml} / \mathrm{kg} / \mathrm{min} ; 60.1 \pm 4.9 \mathrm{ml} / \mathrm{kg}$ / min). Así mismo, Cabrera-Hernández et al. (2018) estudio el VO2max en jugadores profesionales de fútbol con una edad en promedio 18.4 \pm 1 (YYIR1: directo: $54.9 \pm 6.6 \mathrm{ml} / \mathrm{kg} / \mathrm{min}$; indirecto: $56.9 \pm 1.6 \mathrm{ml} / \mathrm{kg}$ / min; y banda sin fin, directo: $55.5 \pm 4.4$ indirecto: 60.00 \pm 3.9); lo mismo describió Le Gall et al. (2010) del V02max mediante un test Vam-Eval en jugadores internacionales de 16 años de edad $(62.4 \pm 2.7 \mathrm{ml} /$ $\mathrm{min} / \mathrm{kg})$, profesionales $(62.2 \pm 3.2 \mathrm{ml} / \mathrm{min} / \mathrm{kg})$ y amateur $(61.7 \pm 3.7 \mathrm{ml} / \mathrm{min} / \mathrm{kg})$; Gil et al. (2010) evaluaron a un grupo de futbolistas en diferentes categorías y posiciones con respecto al VO2max relativo y sus resultados fueron, en delanteros 62.4 \pm 10.76 , medios 57.71 . \pm 9.91, defensas 58.55 \pm 9.48), mientras que Calahorro et al. (2012) obtuvieron en un estudio similar para defensas $61.75 \pm 2.29$, para mediocampistas 62.26 \pm 1.99 y para delanteros $61.97 \pm 2.33$; por su parte Slimani et al. (2019) han analizado el perfil de futbolistas de acuerdo a la edad, posición y el nivel de competencia, así como por sus características antropométricas y fisiológicas; en su trabajo delVO2max muestran rangos a partir de 59.2 y hasta $66.66 \mathrm{ml} / \mathrm{kg} / \mathrm{min}$ para futbolistas profesionales y de $58.8 \mathrm{a} 61.7 \mathrm{ml} / \mathrm{kg} / \mathrm{min}$ para amateurs.

Al comparar nuestro trabajo con otras investigaciones encontramos que mostraron mayor $\mathrm{VO} 2 \mathrm{max}$ al encontrado por nosotros. Por ejemplo, Chamari et al. (2005) evaluó antes y después elVO2max de 8 semanas evidenciando diferencias significativas $(65.3 \pm 5.0 \mathrm{ml} /$ $\mathrm{kg} / \mathrm{min}$ vs $70.7 \pm 4.3 \mathrm{ml} / \mathrm{kg} / \mathrm{min}$ ) en jóvenes futbolistas menores de 15 años; así mismo, Tahara et al. (2006) indicó el promedio del VO2max $(61.4 \mathrm{ml} / \mathrm{kg} / \mathrm{min})$ en 
jugadores japoneses juveniles de fútbol en laboratorio por medio de una banda sin fin.

\section{Efectos en la distancia recorrida}

En nuestro estudio la distancia recorrida por los jugadores juveniles después del IT fue de 1,593 \pm 13.21 $\mathrm{m}$. Estos valores son muy semejantes a los obtenidos por otros investigadores (Zagatto et al., 2013, 2015; León et al., 2015). Por ejemplo, Zagatto et al. (2013) indicaron que la distancia recorrida en el HT por jóvenes futbolistas brasileños de $17 \pm 1$ años con regular entrenamiento sistemático fue de 1,458 $\pm 49.6 \mathrm{~m}$. Por su parte, Zagatto et al. (2015) indicaron que en el test de HT jugadores profesionales de Brasil recorrieron en promedio una distancia de 1,442.4 $\pm 30 \mathrm{~m}$. Por su parte, León et al. (2015) reportaron, usando HT, en jugadores de fútbol que comprendieron una edad de 18.9 \pm 1.8 años, que recorrieron $1,597 \pm 143.2 \mathrm{~m}$. Sin embargo, Castagna et al., (2010) señalaron que la distancia que obtuvieron en el HT fue de 1,059 $\pm 191 \mathrm{~m}$ (666 m mínimo, 1,280 m máximo). En este último caso dicha distancia fue menor a la distancia recorrida en nuestro estudio, esto podría ser debido a la edad de los sujetos muestreados (que eran menores de 15 años de edad) o posiblemente a la forma física de los futbolistas. Ahora bien, Chamari et al. (2005) reportaron una distancia de $1,942 \pm 154 \mathrm{~m}$ para jugadores tunecinos de 15 años de edad, que es mayor a la que nosotros describimos. Sin embargo, el estudio de Chamari et al. (2005) encontró que el incremento del porcentaje de la distancia recorrido en el HT fue de 10 por ciento después de ocho semanas de IT; algo semejante a nuestro nueve por ciento de mejora después del IT. Esto indica que incluso con diferencias de edad y de la forma física de los sujetos, existe claramente un efecto positivo de la IT en los jugadores de fútbol juveniles en la distancia recorrida en el HT.

\section{Conclusiones}

En este estudio observamos que ocho semanas de IT aumentaron el rendimiento de los futbolistas juveniles mexicanos analizados, incrementando su MAS, VO2max, distancia recorrida y frecuencia cardiaca basal; Nuestros resultados son similares a los encontrados por otros autores que usaron una aplicación del IT parecido al nuestro, tanto en el número de semanas de aplicación y cargas aeróbicas específicas. Sin embargo, los resultados de otras investigaciones mostraron un menor o mayor efecto del IT en las variables que investigamos.
En el futuro sería conveniente investigar el porqué de estas diferencias, enfocándose en la aplicación de la carga externa e interna del IT, el nivel de competitividad del equipo (local, estatal, nacional o internacional) y el efecto del nivel de capacidad aeróbica inicial de los jugadores.

\section{Referencias}

Ali, A. (2011). Measuring soccer skill performance: a review. Scandinavian journal of medicine \& science in sports, 21(2), 170-183. doi:10.1111/j.16000838.2010.01256.x

Aziz, A, R., Mukherjee, S., Chia, M.Y. H. \& Tech, K, C. (2008). Validity of the running repeated sprint ability among test playing positions and level of competitiveness in training soccer players. Int J Sport Med, 29 (10), 833838. doi:10.1055/s-2008-1038410

Aziz, A. R., Tan, F. H., \& Teh, K. C. (2005). A pilot study comparing two field tests with the treadmill run test in soccer players. Journal of sports science \& medicine, 4(2), $105-112$.

Bangsbo, J., Nørregaard, L., \& Thorsoe, F. (1991). Activity profile of competition soccer. Canadian journal of sport sciences, 16(2), 110-116.

Barrera, J., Contreras, L. M.V., Cid, F. M., \& Sarmento, H. (2021). Análisis de los componentes físicos y antropométricos de jóvenes futbolistas chilenos desde la categoría Sub-13 a Sub-19. Retos: nuevas tendencias en educación física, deporte y recreación, (39), 547-555. doi: 10.47197/retos.v0i39.79537

Barrero, A, M., Gutiérrez, I. M., \& Prieto, M. F. (2021). Análisis del modelo de juego en un equipo de futbol profesional de la Bundesliga de Alemania. Estudio de caso. Retos, 39, 629-634. doi.org/10.47197/ retos.v0i39.79923

Berthoin, S., Baquet, G., Rabita, J., \& Blondel, N. (1999). Validity of the Universite de MontrealTrackTest to assess the velocity associated with peak oxygen uptake for adolescents. Journal of sports medicine and physical fitness, 39(2), 107-112.

Billat, L. V., \& Koralsztein, J. P. (1996). Significance of the velocity at $\mathrm{VO} 2 \mathrm{max}$ and time to exhaustion at this velocity. Sports Medicine, 22(2), 90-108. doi:10.2165/ 00007256-199622020-00004

Billat, V., Bernard, O., Pinoteau, J., Petit, B., \& Koralsztein, J. P. (1994). Time to exhaustion atVO2max and lactate steady state velocity in sub elite long-distance runners. Archives internationales de physiologie, de biochimie et de biophysique, 102(3), 215-219. doi: 10.3109/ 


\section{1}

Billat, V., Renoux, J. C., Pinoteau, J., Petit, B., \& Koralsztein, J. P. (1995). Times to exhaustion at 90,100 and 105\% of velocity atVO2 max (Maximal aerobic speed) and critical speed in elite long-distance runners. Archives of physiology and biochemistry, 103(2), 129-135. doi:10.3109/ 13813459409007541

Borg, G. (1970). Perceived exertion as an indicator of somatic stress. Scandinavian journal of rehabilitation medicine, 2(2), 392-398.

Bravo, D. F., Impellizzeri, F. M., Rampinini, E., Castagna, C., Bishop, D., \& Wisloff, U. (2008). Sprint vs. interval training in football. International journal of sports medicine, 29(8), 668-674. doi:10.1055/s-2007-989371

Buchheit, M., \& Mendez-Villanueva, A. (2013). Supramaximal intermittent running performance in relation to age and locomotor profile in highly-trained young soccer players. Journal of sports sciences, 31(13), 1402-1411. doi:10.1080/02640414.2013.792947

Buchheit, M., Mendez-Villanueva, A., Quod, M. J., Poulos, N., \& Bourdon,P. (2010a). Determinants of the variability of heart rate measures during a competitive period in young soccer players. European journal of applied physiology, 109(5), 869-878. doi:10.1007/s00421-0101422-x

Buchheit, M., Mendez-Villanueva, A., Simpson, B. M., \& Bourdon, P. C. (2010b). Matchrunning performance and fitness in youth soccer. international journal of sports medicine, 31(11), 818-825. doi:10.1055/s-0030-1262838

Cabrera-Hernández, M. A., Tafur-Tascon, L. J., Cohen, D. D., García-Corzo, S. A., Quiñonez-Sánchez, A., PoveaCombariza, C., \& Tejada-Rojas, C. X. (2018). Concordance between the indirect VO2max value estimated through the distance in Yo-Yo intermittent recovery test level 1 and the direct measurement during a treadmill protocol test in elite youth soccer players. Journal of Human sport \& Exercise, 13(2) 401-412. doi:10.14198/jhse.2018.13.Proc2.24

Calahorro, F., Zagalaz, M. L., Lara, A. J., \& Torres-Luque, G. (2012). Análisis de la condición física en jóvenes jugadores de fútbol en función de la categoría de formación y del puesto específico. Apunts. Educación fisica y deportes, 109(3), 54-62. doi: 10.5672/apunts.2014 0983.es.(2012/3).109.05

Carbonell, A., Aparicio, V., \& Delgado, M. (2009). Valoración de la condición física en futbolistas de categoría cadete. Revista Kronos, 8(15). 101-106 doi:10.14198/ jhse.2018.13.Proc2.24

Carminatti, L. J., Possamai, C. A., De Moraes, M., Da Silva, J. F., De Lucas, R. D., Dittrich, N., \& Guglielmo, L.
G. (2013). Intermittent versus continuous incremental field tests: are maximal variables interchangeable? Journal of sports science \& medicine, 12(1), $165-170$

Castagna, C., Impellizzeri, F., Cecchini, E., Rampinini, E., \& Alvarez, J. C. B. (2009). Effects of intermittentendurance fitness on match performance in young male soccer players. The Journal of Strength \& Conditioning Research, 27(3), 1954-1959. doi: 10.1519/ JSC.0b013e3181b7f743

Castagna, C., Manzi, V., Impellizzeri, F., Weston, M., \& Alvarez, J. C. B. (2010). Relationship between endurance field tests and match performance in young soccer players. The Journal of Strength \& Conditioning Research, 24(12), 3227-3233. doi:10.1519/JSC.0b013e3181e72709

Castañer, M., Barreira, D., Camerino, O., Anguera, M. T., Fernandes, T., \& Hileno, R. (2017). Mastery in goal scoring,T-pattern detection, and polar coordinate analysis of motor skills used by Lionel Messi and Cristiano Ronaldo. Frontiers in psychology, 8(741), 1-18 doi: 10.3389/fpsyg.2017.00741

Castro L, E., Arguello Y, P., Sánchez, I, A., Jazmín, A., \& Melo P, J. (2021) Relación entre marcadores dermatoglíficos y el perfil morfofuncional en futbolistas de Bogotá, Colombia. Retos (41), 182-190. doi.org/ 10.47197/retos.v0i41.83032

Cazorla, G. (1990) Field tests to evaluate aerobic capacity and maximal aerobic speed. In: Proceedings of the International Symposium of Guadeloupe. Edts: Actshng and Areaps. 151-173.

Chamari, K., Hachana, Y., Kaouech, F., Jeddi, R., MoussaChamari, I., \& Wisløff, U. (2005). Endurance training and testing with the ball in young elite soccer players. British journal of sports medicine, 39(1), 24-28. doi.org/ 10.1136/bjsm.2003.009985

Crawley, M. (1993). GLIM for ecologist. London: Blackwell Scientific Publications.

Cunha, G., Lorenzi, T., Sapata, K., Lopes, A. L., Gaya, A. C., \& Oliveira, Á. (2011). Effect of biological maturation on maximal oxygen uptake and ventilatory thresholds in soccer players: an allometric approach. Journal of sports sciences, 29(10), 1029-1039. doi:10.1080/ 02640414.2011 .570775

Di Prampero, P. (1986) The anaerobic threshold concept: a critical evaluation. Adv. Cardiol 35, 24-34.

Dupont, G., Akakpo, K., \& Berthoin, S. (2004). The effect of in-season, high-intensity interval training in soccer players. The Journal of Strength \& Conditioning Research, 18(3), 584-589. doi:10.1519/15334287(2004)18<584:TEOIHI>2.0.CO;2 
Dupont, G., Defontaine, M., Bosquet, L., Blondel, N., Moalla, W., \& Berthoin, S. (2008). Yo-Yo intermittent recovery test versus the Universite de Montreal Track Test: relation with a high-intensity intermittent exercise. Journal of Science and Medicine in Sport, 13(1), 146-150. doi:10.1016/j.jsams.200810.10007

Echavarría, S.G., Arango, M.S., Arango, S.S., Sánchez, W. G.V., \& Ramos J.A.E. (2021) Nivel de adaptación al contexto táctico en futbolistas juveniles. Retos. (41), 237 246. 10.47197/retos.v0i41.83509

Ferreira, E. C., Andrade, V. L., Campos, E. Z., Tacioli, F., Freire, A. O., \& Lourenção, A. (2011). Determination of maximal lactate steady state in soccer specific test: a pilot study. Int J ExercSci, 7, S40.

Fox, E. L. Bowers, R. W., \& Foss, M. L. (1997). The physiological basis for exercise and sport. Dubeque, Iowa: Brown and Benchmark.

Gil, S. M., Gil, J., Ruiz, F., Irazusta, A., \& Irazusta, J. (2010). Anthropometrical characteristics and somatotype of young soccer players and their comparison with the general population. Biology of Sport, 27(1), 17-24. doi: $10.5604 / 20831862.906762$

Gomez-Piqueras, P., Malavés, R. A., \& López, V. F. (2010). Seguimiento longitudinal de la evolución en la condición aeróbica en jóvenes futbolistas. Apunts. Medicina de l'Esport, 45(168), 227-234. doi:10.1016/ j.apunts.2010.03.002

Hammer, O., Harper, D.T.A., \& Ryan, P.D. (2001). PAST: paleontological Statistical Software package for education and data analysis. Paleontología Electrónica: 4(1): 9

Helgerud, J., Engen, L. C., Wisloff, U., \& Hoff, J. (2001). Aerobic endurance training improves soccer performance. Medicine and science in sports and exercise, 33(11), 1925 1931. doi:10.1097/00005768-200111000-00019

Hoff, J. (2005). Training and testing physical capacities for elite soccer players. Journal of sports sciences, 23(6), 573582. doi:10.108/02640410400021252

Hoff, J., Wisløff, U., Engen, L. C., Kemi, O. J., \& Helgerud, J. (2002). Soccer specific aerobic endurance training. British journal of sports medicine, 36(3), 218-221. doi:10.1136/bjsm.36.3.218

Iaia, F. M., Ermanno, R., \& Bangsbo, J. (2009). High-intensity training in football. International journal of sports physiology and performance, 4(3), 291-306. doi: 10.1123/ijspp.4.3.291

Impellizzeri, F. M., Marcora, S. M., Castagna, C., Reilly, T., Sassi,A., laia., \& F. M. Rampinini, E. (2006). Physiological and performance effects of generic versus specific aerobic training in soccer players. International journal of sports medicine, 27(06), 483-492. doi:10.1055/s-2005-865839

Kemi, O. J., Hoff, J., Engen, L. C., Helgerud, J., \&Wisloff,
U. (2003). Soccer specific testing of maximal oxygen uptake. Journal of Sports Medicine and Physical Fitness, 43(2), 139-144.

Kuipers, H., Verstappen, F,T. J., Keizer, H, A., Geurten, P., \&Van Kranenburg, G. (1985) Variability of aerobic performance in the laboratory and its physiologic correlates. Int J Sports Med. 6(4):197-201. doi:10.1055/s-20081025839.

Le Gall, F., Carling, C., Williams, M., \& Reilly, T. (2010). Anthropometric and fitness characteristics of international, professional and amateur male graduate soccer players from an elite youth academy. Journal of science and medicine in sport, 13(1), 90-95. doi/ 10.1016/ j.jsams.2008.07.004

Léger, L., \& Boucher, R. (1980). An indirect continuous running multistage field test: the Universite de Montreal track test. Can.J.Appl. Sport. Sci, 5, 77-84.

Léger, L., \& Mecier, D. (1984). Gross energy cost of horizontal treadmill and track running. Sports medicine, 1(4), 270-277.

León, H. H., Ramírez, J. F., Sánchez, A., Salazar, J. D., Orjuela, L., \& Anzola, S. V. (2015). Comparison of maximum lactate between course navette test and hoff test in soccer players at 2600 meters above sea level. Journal human sport \& exercise, 16(1), 104-112. doi: 10.14198/jhse.2015.101.09

Los Arcos, A., Vázquez, J. S., Villagra, F., Martín, J., Lerga, J., Sánchez, F., Berto, J., \& \& Zulueta, J. J. (2019). Assessment of the maximal aerobic speed in young elite soccer players: Université de Montréal Track Test (UM-TT) vs. treadmill test. Science \& Sports, 34(4), 267271. doi: 10.1016/j.scispo.2019.03.010

Loures, J. P., Chamari, K., Ferreira, E. C., Campos, E. Z., Zagatto, A. M., Milioni, F., Da Silva, RS., \& Papoti, M. (2015). Specific determination of maximal lactate steady state in soccer players. The Journal of Strength \& Conditioning Research, 29(1), 101-106. doi: 10.1519/ JSC.0000000000000621

Martínez-Cabrera, F.I., Núñez-Sánchez, F. J., Muñoz-López A., \& De Hoyo M, (2021) Aceleraciones de alta intensidad en el futbol. ¿Por qué es importante el método de evaluación? Retos, (39), 750-754. doi.org/10.47197/ retos.v0i39.82281

McMillan,K., Helgerud, J., Macdonald, R., \& Hoff, J. (2005). Physiological adaptations to soccer specific endurance training in professional youth soccer players. British journal of sports medicine, 39(5), 273-27. doi:10.1136/ bjsm.2004.012526

Mendez-Villanueva, A., Buchheit, M., Kuitunen, S., Poon, T. K., Simpson, B. , \& Peltola, E. (2010). Is the relationship 
between sprinting and maximal aerobic speeds in young soccer players affected by maturation? Pediatric exercise science, 22(4), 497-510. doi:10.1123/pes.22.4.497

Mendez-Villanueva, A., Buchheit, M., Simpson, B., \& Bourdon, P. C. (2013). Match play intensity distribution in youth soccer. Int J Sports Med, 34(2), 101-110. doi:10.1055/s-0032-1306323

Nassis, G. P., Geladas, N. D., Soldatos, Y., Sotiropoulos, A., Bekris, V., \& Souglis, A. (2010). Relationship between the 20-m multistage shuttle run test and 2 soccer-specific field tests for the assessment of aerobic fitness in adult semi-professional soccer players. The Journal of Strength \&Conditioning Research, 24(10), 2693-2697. doi: 10.1519/ JSC.0b013e3181bf0471

Ortiz-Pulido, R., Ortiz-Pulido, R., \& Ortiz-Pulido, R. (2018). Consumo máximo de oxígeno en mexicanos universitarios: correlación entre cinco test predictivos. Revista Internacional de Medicina y Ciencias de la Actividad Física y del Deporte, 18(71), 521-535. doi:10.15366/rimcafd2018.71.008

Padilla, S., Bourdin, M., Barthelemy, J. C., \& Lacour, J. R. (1992). Physiological correlates of middle-distance running performance. European journal of applied physiology and occupational physiology, 65(6), 561-566. doi:10.1007/ BF00602366

Paul, D. J., Marques, J. B., \& Nassis, G. P. (2019). The effect of a concentrated period of soccer-specific fitness training with small-sided games on physical fitness in youth players. J Sports Med Phys Fitness, 59(6), 962-968. doi:10.23736/S0022-4707.18.08547-X

Rampinini, E., Bishop, D., Marcora, S. M., Bravo, D. F., Sassi, R., \& Impellizzeri, F. M. (2007). Validity of simple field tests as indicators of match-related physical performance in top-level professional soccer players. International journal of sports medicine, 28(3), 228-235. doi:10.1055/s2006-924340

Rienzi, E., Drust, B., Reilly,T., Carter, J.E. X. L., \& Martin, A. (2000). Investigation of anthropometric and workrate profiles of elite South American international soccer players. Journal of sports medicine and physical fitness, 40(2), 162-169.

Santos-Silva, P. R., Fonseca, A. J., Castro, A. W. D., Greve, J. M. D. A., \& Hernandez, A. J. (2007). Reproducibility of maximum aerobic power (VO2max) among soccer players using a modified heck protocol. Clinics, 62(4), 391-396. doi:10.1590/S1807-59322007000400004

Sassi, R., Reilly, T., \& Impellizzeri, F. (2005). A comparison of small-side games and interval training in elite professional soccer players. Science and football V. Oxon: Routledge, 352-354.
Slimani, M.,Znazen, H., Miarka, B., \& Bragazzi, N. L. (2019). Maximum oxygen uptake of male soccer players according to their competitive level, playing position and age group: implication from a network metaanalysis. Journal of human kinetics, 66(1), 233-245. doi:10.2478/hukin-2018-0060

Sousa, H, Gouveia, É. R., Marques, A., Sarmento, H., Caldeira, R., Freitas, R., Lopes, H., Prudente, J., \& Ihle,A. (2021). El efecto de los juegos reducidos de futbol equilibrados y desequilibrados en la calificación del esfuerzo percibido en los jugadores juveniles. Retos, (41), 440-446. doi.org/10.47197/retos.v0i41.83091

Stølen, T., Chamari, K., Castagna, C., \& Wisløff, U. (2005). Physiology of soccer. Sports medicine, 35(6), 501-536. doi:10.2165/00007256-200535060-00004

Tahara,Y., Moji, K., Tsunawake, N., Fukuda, R., Nakayama, M., Nakagaichi, M., Komine, T., Aoyagi, K., \& Aoyagi, K. (2006). Physique, body composition and maximum oxygen consumption of selected soccer players of Kunimi High School, Nagasaki, Japan. Journal of physiological anthropology, 25(4), 291-297.doi:10.2114/jpa2.25.291

Zagatto, A. M., Miyagi, W. E., Sakugawa, R. L., \& Papoti, M. (2013). Use of maximal running distance performed on Hoff test for anaerobic threshold prediction in soccer. Revista Brasileira de Medicina do Esporte, 19(4), 267-270. doi:10.1590/S1517-86922013000400008

Zagatto, A. M., Miyagi, W. E., Sakugawa, R. L., \& Papoti, M. (2015). Correlation between Hoff test performance, body composition and aerobic and anaerobic fitness in professional soccer players. Sport Sciences for Health, 11(1), 73-79. doi:10.1007/s11332-014-0210-0

Zagatto, A. M., Papoti, M., Da Silva, A. S. R., Barbieri, R. A., Campos, E.Z., Ferreira, E. C., \& Chamari, K. (2016). The Hoff circuit test is more specific than an incremental treadmill test to assess endurance with the ball in youth soccer players. Biology of sport, 3(33), 263-268. doi: 10.5604/20831862.1201913

Zouhal, H., LeMoal, E.,Wong, D. P., Benounis, O., Castagna, C., Duluc, C., Owen, L., \& Drust, B. (2013). Physiological responses of general vs. specific aerobic endurance exercises in soccer. Asian journal of sports medicine, 4(3), 213-220. doi:10.5812/asjsm.34285

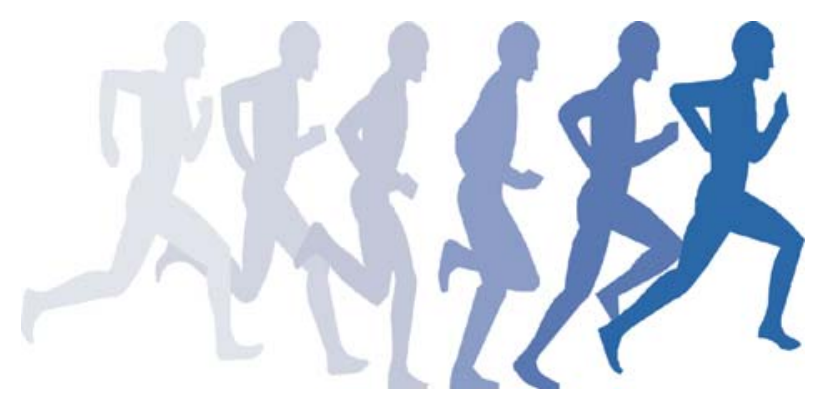

